\title{
Probabilistic route reliability with distance
}

\begin{abstract}
In mobile ad hoc networks, node mobility causes frequent link failures, thus invalidating the routes containing those links. This leads the frequent operation of route reconstruction that consumes lots of the network resources and the energy of nodes. Many efforts have been made to design reliable routing protocols that discover long lifetime routes. In these protocols by using reliability metrics for route selection, more reliable routes are discovered. In this paper, we review two mostly used network layer reliability metrics; ñRoute Expiration Timeò and ñProbabilistic Route Reliabilityò and propose a new one; ñProbabilistic Route Reliability with Distanceò Afterwards, the efficiency of these reliability metrics is evaluated by simulation experiments in different network conditions. The focus was concentrated on the number of route reconstructions as the performance metric. Simulation results show that by using all these three reliability metrics, number of route reconstructions is reduced and for the proposed one, the results are better than the others.
\end{abstract}

Keyword: Mobile ad hoc network; Reliable routing; Long lifetime route 